\title{
Multisensory anatomical pathways
}

\author{
C. Cappe ${ }^{\mathrm{a}, *}$, E.M. Rouiller ${ }^{\mathrm{b}}$, P. Barone ${ }^{\mathrm{c}, *}$ \\ ${ }^{a}$ The Functional Electrical Neuroimaging Laboratory, Neuropsychology and Neurorehabilitation Service and Radiology Service, \\ Centre Hospitalier Universitaire Vaudois and University of Lausanne, rue du Bugnon 46, 1011 Lausanne, Switzerland \\ ${ }^{\mathrm{b}}$ Unit of Physiology and Program in Neurosciences, Department of Medicine, Faculty of Sciences, University of Fribourg, Rue du Musée 5, CH-1700 Fribourg, Switzerland \\ ${ }^{\mathrm{c}}$ Centre de Recherche Cerveau et Cognition (UMR 5549) CNRS, Université Paul Sabatier Toulouse 3, Faculté de Médecine de Rangueil, 31062 Toulouse Cedex 9, France
}

\section{Introduction}

The cerebral network for multisensory integration was classically viewed as a hierarchically converging system in which the different sensory channels were merging in the polysensory associative areas of the frontal, temporal or parietal lobes. In support of this view was the absence of strong anatomical links between the areas of different modalities at the level of the first stages of sensory processing (see Jones and Powell, 1970). Recent anatomical and electrophysiological studies in non-human primates as well as human functional brain studies led to a reappraisal of this

Abbreviations: CAC, caudal auditory cortex; CL, central lateral nucleus; CM, centre median nucleus; CT, corticothalamic; FB, feedback; FF, feedforward; FSTd, dorsal fundus of the superior temporal area; FSTv, ventral fundus of the superior temporal area; IPS, intraparietal sulcus; LIP, lateral intraparietal area; LP, lateral posterior nucleus; MD, mediodorsal nucleus; MGN, medial geniculate nucleus; MT, medial temporal area; MTc, caudal part of MT; PMd, dorsal premotor cortex; PMv, ventral premotor cortex; PuL, lateral pulvinar; PuM, medial pulvinar; RAC, rostral auditory cortex; Ri, retro-insular cortex; SC, superior colliculus; STP, superior temporal plane; STS, superior temporal sulcus; TC, thalamocortical; VA, ventral anterior nucleus; VIP, ventral intraparietal area; VLpd, ventral lateral posterior nucleus, dorsal division; VPL, ventral posterior lateral nucleus

* Corresponding authors. Tel.: +41 2131475 12; fax: +41 213141319 (C. Cappe); tel.: +33 5621737 79; fax: +33 562172809 (P. Barone).

E-mail addresses: celine.cappe@chuv.ch (C. Cappe), eric.rouiller@unifr.ch (E.M. Rouiller), pascal.barone@cerco.ups-tlse.fr (P. Barone). concept (see Ghazanfar and Schroeder, 2006; Driver and Noesselt, 2008 for recent reviews) and highlight that the mechanisms for multisensory interplay are believed to include several levels of brain processing, from the thalamus to the primary sensory areas and higher stages of sensory processing. Such an increase in the diversity of the pathways by which multisensory interactions can occur is probably reflecting an adaptive mechanism by which individual perceptual or sensory-motor situations involve a specific multisensory network. We describe in this review connections in the brain that may represent the support for early multisensory integration, such as cortico-cortical connections and connections between the thalamus and the cortex.

\section{Heteromodal connections: connections between different sensory areas}

The convergence of different sensory information channels in polysensory cortical areas is now clearly established and will not be detailed in the present report. Most areas in the parietal, temporal, or frontal regions of primates have connection patterns that relate them to more than one sensory modality. This is particularly the case for the areas buried in the intraparietal sulcus (LIP, VIP) which present anatomical links with cortical areas of different sensory modalities (Lewis and Van Essen, 2000). For instance, in the 
monkey, the parietal area VIP receives inputs from visual, auditory, somatosensory, vestibular, motor and polysensory areas (Duhamel et al., 1998) that confer typical multimodal responses to the VIP neurons (Bremmer et al., 2002; Schlack et al., 2005; Avillac et al., 2007). Similarly, the connectivity pattern of the LIP (Andersen et al., 1990; Blatt et al., 1990; Lewis and Van Essen, 2000) is consistent with the multisensory properties reported for LIP neurons (Cohen et al., 2005; Russ et al., 2006; Gottlieb, 2007). The ventral part of LIP connects with areas dealing with spatial information (visual area MT and the auditory caudiomedial area; Andersen et al., 1997), as well as with the frontal eye field (Schall et al., 1995), whereas the dorsal part of LIP is connected with areas responsible for the processing of visual information related to the form of objects in the inferotemporal cortex (ventral "what" visual pathway). In parallel, the temporal region of the superior temporal sulcus (STS) is connected with the visual occipital cortices (Seltzer and Pandya, 1994) and with the secondary auditory area (area 22 of Brodmann; Pandya and Seltzer, 1982) providing the multimodal properties of neurons in area STP (Bruce et al., 1981; Baylis et al., 1987; Hikosaka et al., 1988). Along the same line, the prefrontal cortex, playing a role in temporal integration, receives projections from the auditory and the visual cortices (e.g. Gaffan and Harrison, 1991; Romanski et al., 1999; Fuster et al., 2000). Still in the monkey, a network of inter-connected multisensory areas playing a role in the perception of visual, auditory and tactile stimuli presented in a part of space close to the body was described, involving the putamen, the premotor cortex, VIP and the parietal area 7b (Graziano and Gross, 1998; Graziano, 2001).

The notion that multisensory integration is restricted to higherorder areas has recently been challenged by human and animal studies that have revealed that crossmodal interactions can occur in unisensory areas at very low levels of cortical processing (Buchel et al., 1998; Calvert et al., 1999, 2001; Macaluso et al., 2000; Schroeder et al., 2001; Amedi et al., 2002; Ghazanfar et al., 2005; Kriegstein et al., 2005; Miller and D'Esposito, 2005; Watkins et al., 2006; Martuzzi et al., 2007; Kayser et al., 2007, 2008; Romei et al., 2007, 2008; Wang et al., 2008) and more importantly at very short latencies (Giard and Peronnet, 1999; Foxe et al., 2000; Molholm et al., 2002; Murray et al., 2005; Senkowski et al., 2007; Sperdin et al., 2009). Such a fast timing of multisensory interactions rule out an origin in the multisensory areas mediated through backward projections, and instead favor direct heteromodal connections. Using anatomical tracers we have identified a set of heteromodal connections in the monkey that connect directly areas devoted to different sensory modalities (see Fig. 1A and B).

\subsection{Primary sensory areas receive non-specific inputs}

Although somewhat surprising, there is evidence for the existence of multisensory neurons in areas traditionally considered as unisensory, such as the visual cortex (between areas 17 and 18a) in the rat (Barth et al., 1995) and in the auditory cortex of the monkey (Watanabe and Iwai, 1991; Schroeder et al., 2001; Cappe et al., 2007b; Kayser et al., 2008) and of the ferret (Bizley et al., 2007; Bizley and King, 2008). In addition, previous studies have shown the existence of scattered projections of the auditory cortex towards the visual area 18 in the rat and the cat (Miller and Vogt, 1984; Innocenti et al., 1988). However, no similar result had been observed in the monkey. But, very recently, the use of anterograde and retrograde tracers in the monkey made it possible to highlight direct projections of the auditory cortex to the primary visual cortex V1 (Falchier et al., 2002), as well as of the associative auditory cortex to the primary and secondary visual areas (Rockland and Ojima, 2003) (Fig. 1). These direct connections arise in their majority from the parabelt auditory area located on the supratemporal gyrus (Falchier et al., 2002). We have estimated that about a third of the auditory projections originate from the anterior bank of the lateral sulcus, but only a small fraction of these projecting neurons are located in the primary auditory cortex. Such results are similar to those observed in the cat (Hall and Lomber, 2008), in which the direct connections between the two primary sensory areas (from A1 to A17) represent only a small percentage (about $5 \%$ ) of the auditory inputs to the primary visual area. In the monkey we did not find that the auditory projection to V1 was reciprocal, but a recent anatomical study showed projections from extrastriate visual area V2 to the auditory area Tpt (temporoparietal temporal area) in the superior temporal gyrus (Nascimento-Silva et al., 2005), as well as to the auditory belt and parabelt (see Falchier et al. in this issue), suggesting that early visual processing can influence the auditory cortex (Fig. 1). Further, in the same neuroanatomical study, we found that area STP located in the anterior bank of the STS sends projections to V1 (Fig. 1A). Because this visual area also contains a large proportion of auditory and somatosensory neurons (Bruce et al., 1981; Hikosaka et al., 1988 ), this projection can also provide non-visual information to the primary visual cortex.

The existence of heteromodal connections at early stages has been confirmed in the New World monkey, the marmoset (Cappe and Barone, 2005) (Fig. 1A and B). We found direct projections from visual areas to the primary auditory cortex and to the primary somatosensory cortex. We also demonstrated the existence of connections between the primary somatosensory cortex and the primary auditory cortex. More precisely, these visuo-somatosensory projections originated from visual areas (the ventral and dorsal fundus of the superior temporal area - FSTv and FSTd - and middle temporal crescent (MTc)) and were sent towards areas $1 / 3 \mathrm{~b}$. Somatosensory projections to the auditory cortex arose from the area S2 and the anterior bank of the lateral sulcus. Finally, a visuo-auditory projection arose from an area anterior to the superior temporal sulcus (STS) towards the auditory core. The heteromodal connections between the primary somatosensory cortex and the primary auditory cortex were also reported in the gerbils (Budinger et al., 2006). In marmosets, projections from the retroinsular area of the somatosensory cortex to the caudiomedial belt auditory area were also observed (de la Mothe et al., 2006a) in line with a similar observation in the Old World monkey (Smiley et al., 2007).

In all cases, these heteromodal connections were described as not very dense, representing only few hundred projecting neurons. While this low strength of projection could be interpreted as of low functional impact, it is important to consider that these projections are observed following small size injections of retrogradely transported dyes covering only a restricted portion of the cortex. For example, we estimated that these heteromodal projections constitute only a few percent of the total cortical inputs of the somatosensory cortex of the marmoset. However, the non-visual projections to the peripheral $\mathrm{V} 1$ can be as large as the projection arising from the motion visual area MT/V5 (Falchier et al., 2002), which is known to exert an influential impact on V1 responses (Hupé et al., 1998). In addition, all of these heteromodal connections do not consist in scattered projecting neurons, but rather have a topological organization (Batardiere et al., 1998) with a relatively compact projection zones probably linked to cortical functional modules. For example, in the cat, the auditory projection from area PAF is organized with respect to the retinotopic organization of the primary visual cortex (Hall and Lomber, 2008).

In the sensory system of the monkey, the laminar organization of the neurons of origin and of the terminals allows to distinguish feedback (FB) and feedforward (FF) connections (Felleman and Van Essen, 1991). While the role of FF and FB connections is only partially understood (Salin and Bullier, 1995; Bullier, 2006), the nature of these connections has been used to establish a hierarchical relationship between cortical areas (Maunsell and Van Essen, 1983; 

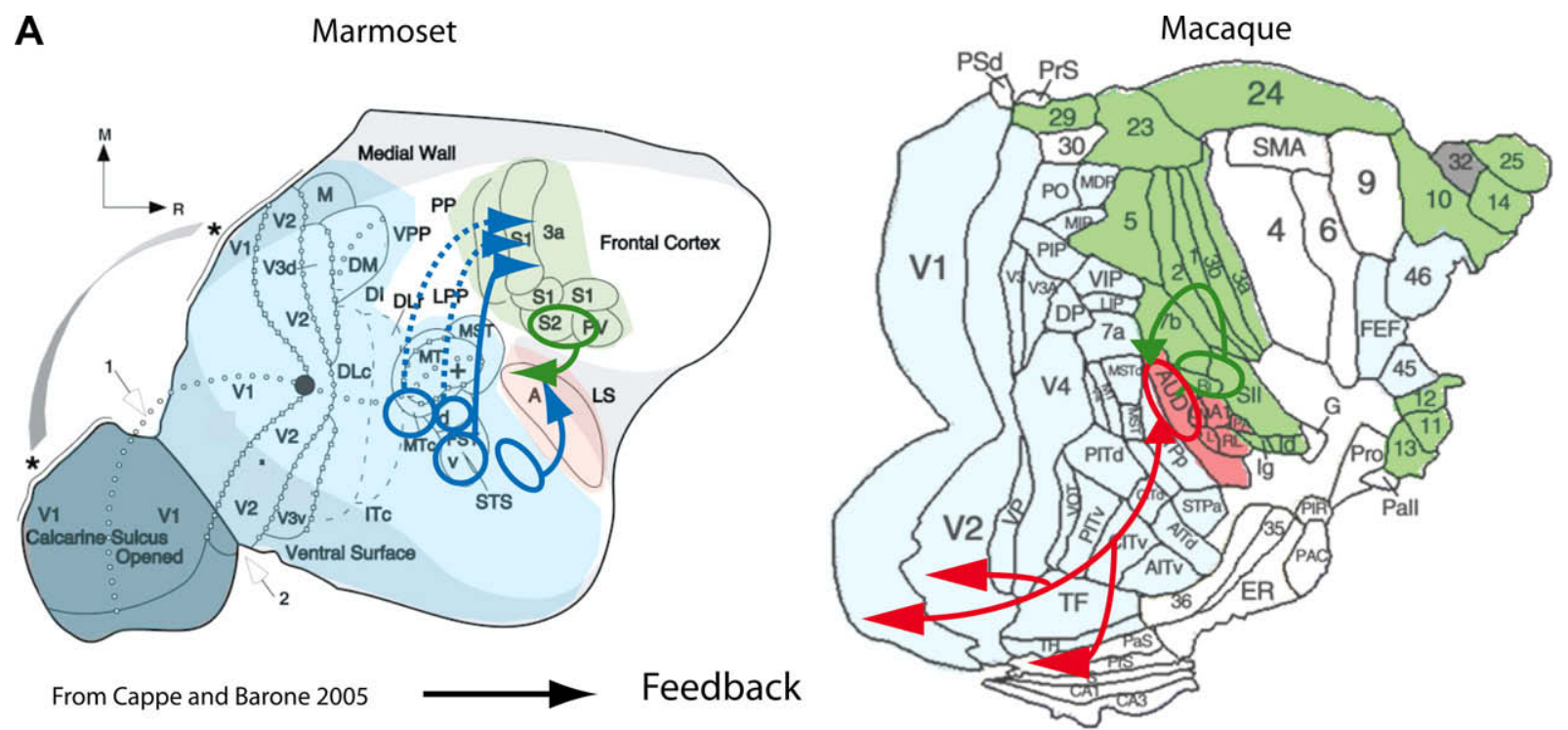

From Cappe and Barone 2005

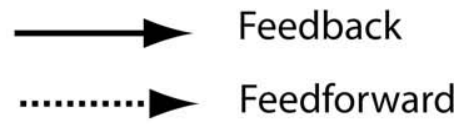

From Falchier et al 2002, 2009

Visual cortical areas

Auditory cortical areas

Somatosensory cortical areas

B

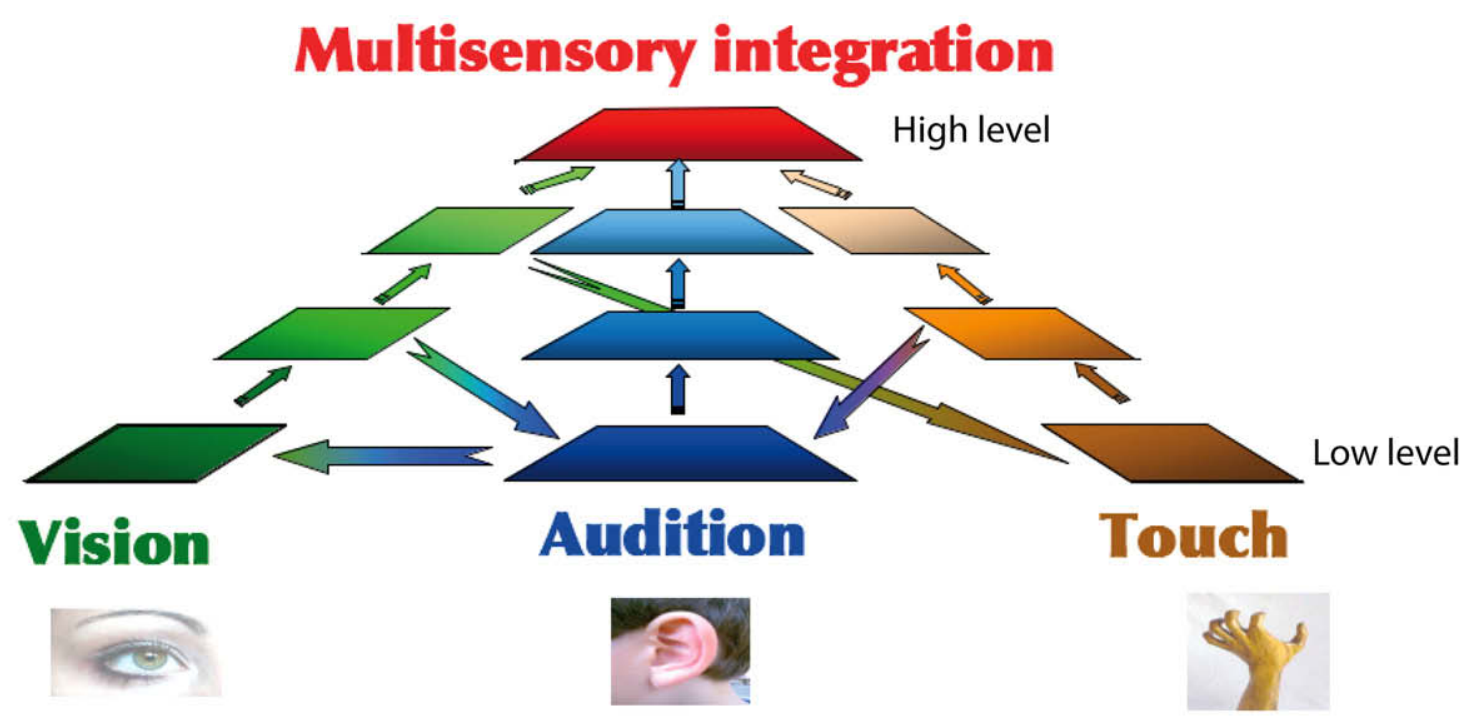

Fig. 1. (A) Flattened representation of the marmoset cortex (left, adapted from Lyon and Kaas, 2001) and the macaque monkey (right, adapted from Felleman and Van Essen 1991) showing some direct cortico-cortical connections between different sensory areas, as derived from the studies indicated below each panel (see also Falchier et al., in this issue). (B) Cortico-cortical connections between different sensory areas at low-level and considered until recently as unisensory areas are represented schematically as transversal arrows between several levels of processing of vision, audition and touch and are integrated in the hierarchical model of sensory processing.

Felleman and Van Essen, 1991) and determine the direction of sensory information transfer. Based on a quantitative analysis of the laminar distribution of retrogradely labeled neurons, the heteromodal connections could be classified as FF or FB type (Cappe and Barone, 2005). Indeed, both the visual projection from STS and somatosensory projection to the primary auditory cortex are of FB type with the majority of labeled cells located in infragranular layers. However, while the visual projections from the FST ventral area to the primary somatosensory cortex show an anatomical profile of FB type, the ones that originate from the visual areas FST dorsal and MTc areas and targeting area 1/3b correspond to projections of FF type (Fig. 1). Lastly, in the marmoset, the projections from polysensory areas of the frontal lobe to the auditory, somatosensory and visual cortices are of FB or FF type, depending on the sensory projection and area to which it projects. This analysis showed that heteromodal connections do not present a unique profile of projections as they were found to be either of FB or FF type (Fig. 1). This is of importance knowing that the FB and FF patterns are usually associated with distinct functional influences on the target neurons (Gonchar and Burkhalter, 1999).

Further, some uncertainty remains on the exact classification of the heteromodal connections in the scheme of the FB/FF anatomical dichotomy. For example, Schroeder and his colleagues (2001) observed that somatosensory responses in the caudal auditory belt 
were initially focused on layer 4 according to the type of stimulation, suggesting that the somatosensory inputs are transmitted via a driving FF type connection. This contradicts the anatomical data which have revealed two sources of somatosensory inputs to the auditory belt: a weak direct projection from S2 (Cappe and Barone, 2005) and a substantial source of afferents located in the retroinsular cortex Ri (de la Mothe et al., 2006a,b; Smiley et al., 2007). In all cases the projections towards the auditory cortex originated from the infragranular layers, an anatomical feature of FB projections. This discrepancy may be due to the fact that the source of somatosensory responses recorded in the auditory cortex is not cortical, but instead originates from non-specific thalamic inputs (Cappe et al., 2009a). It is also possible that the classical anatomical classification of FF and FB projections does not apply to heteromodal projections when it comes to interactions between sensory modalities. This would support the hypothesis according to which the anatomical pattern of the cortico-cortical connections that involve the polysensory areas of the frontal lobe depend on the intrinsic architecture of areas linked together in terms of laminar differentiation (Barbas and Rempel-Clower, 1997; Rempel-Clower and Barbas, 2000). How this rule of a structural dependence about the laminar organization of projections affects heteromodal connections remains to be determined.

\subsection{Specificity of heteromodal connections: ethological role}

In the somatosensory system, there is evidence for the existence of a different connectivity pattern according to the body representation, especially in terms of density of connections between areas (Krubitzer and Kaas, 1990; Huffman and Krubitzer, 2001). In the visual system, density and laminar profile of the connections between visual areas also differ depending on whether they involve the representation of the central or peripheral visual field (Shipp and Zeki, 1989; Kaas and Morel, 1993; Schall et al. 1995; Galletti et al. 2001; Palmer and Rosa, 2006). Our connectivity data show that heteromodal connections are also specific to the sensory representation. We found that the visual projections to areas $1 / 3 \mathrm{~b}$ are restricted to the representation of certain body parts. Some visual projections are targeting selectively the face (MTc) or the arm (FSTd) representations in areas $1 / 3 \mathrm{~b}$. Because both injections sites involved two areas (areas 1 and $3 \mathrm{~b}$ ), we cannot determine whether this reflects the preferential connections to either area. Similarly, auditory and multimodal projections to area V1 are prominent towards the representation of the peripheral visual field (Falchier et al., 2002), and only scattered neurons in the auditory cortex send a projection to foveal V1 (Fig. 1A). The fact that the heteromodal connections are coupling specific sensory representations across modalities probably reflects an adaptive process for behavioral specialization. For example, when comparing across species, it has been inferred that the spatial efficiencies of the auditory and visual modalities are tightly related (Heffner and Heffner, 1992), in such a way that the main function of sound localization is to direct the field of higher visual acuity to a sound source for further analysis. Such complementarities can also be understood at the level of the heteromodal connections between the primary visual and auditory areas. In the primary auditory cortex of the cat, the auditory receptive fields are generally large (Clarey et al., 1995) and for the majority of them, they extend over the pinna axis in the peripheral space (Barone et al., 1996). Considering that only the peripheral visual field representation of $\mathrm{V} 1$ receives significant projections from the auditory cortex, such congruency in the spatial features may serve to facilitate gaze orienting, and consequently the relocation of foveal vision to peripheral locations in the visual field. However, it should also be mentioned that facilitative effects have been observed in humans when stimuli were centrally presented in both auditory and visual modalities (Giard and
Peronnet, 1999; Molholm et al., 2002; Martuzzi et al., 2007). Ethologically, a role in alertness for dangerous stimuli is highly probable, an interpretation that can also be attributed to the visuo-somatosensory projections: the specific link between the FST visual complex and the representation of the face in the somatosensory cortex could contribute to phenomena of avoidance of a "dangerous" stimulus which may hit the body (Cooke and Graziano, 2003, 2004). More recently, there is also evidence for selective interactions between auditory-visual cues signaling approach or looming (Maier et al., 2004, 2008; Cappe et al., 2009b).

\section{Thalamocortical connections}

\subsection{Role of the thalamus}

Although mutisensory integration has been shown to take place essentially in the cerebral cortex and the superior colliculus (Stein and Meredith, 1993), one may consider the possibility of complementary contributions taking place at a subcortical level. In this context, given the extensive connections between the neocortex, the superior colliculus and the thalamus, different authors have proposed that subcortical structures integrate the senses even before the neocortex (Ghazanfar and Schroeder, 2006 for review). The thalamus is a possible candidate considering its strong input-output connections with multiple cortical areas, both sensory and motor.

Along this line, one may take into account that many corticocortical pathways are transmitting information relatively slowly. The corticothalamic projections are usually seen as a specific feedback projection, by which a given cortical area is controlling the thalamic nucleus from which it receives ascending input. However, recent data provide evidence that a separate, highly specialized corticothalamic projection is in a position to provide feedforward projections from one cortical area to other cortical regions via the thalamus (see for review Rouiller and Welker, 2000; Sherman and Guillery, 2002, 2005; Sherman, 2007). In contrast to the feedback corticothalamic projection originating from cortical layer VI, the feedforward corticothalamic projection comes from layer $\mathrm{V}$ and terminates in the thalamus in the form of giant endings, which can ensure highly secure and rapid synaptic transmission (Rouiller and Welker, 2000). Such feedforward corticothalamic projections with giant endings have been demonstrated for many sensory and motor cortical areas (e.g. Darian-Smith et al., 1999; Ojima, 1994; Rockland, 1996; Rouiller and Durif, 2004; Rouiller et al., 1998, 2003; Schwartz et al., 1991; Taktakishvili et al., 2002; Cappe et al., 2007a), representing an anatomical support for fast interactions between distant cortical regions, via the thalamus (see also Feig and Harting, 1998; Sherman and Guillery, 2002; Van Horn and Sherman, 2004). Such cortico-thalamo-cortical routing is thus suitable for multisensory interplay by a convergence of different modalities on the same thalamic nucleus that then in turn makes this integrated information available to a target cortical region, for instance the premotor cortex (PM) or the prefrontal cortex (Pf). The pulvinar nucleus, among other associative thalamic nuclei, is a good candidate to play such an integrative role, based on its connectivity with numerous cortical areas (e.g. Romanski et al., 1997; Hackett et al., 1998; Gutierrez et al., 2000) and on electrophysiological studies (Yirmiya and Hocherman, 1987; Gattass et al., 1979).

This hypothesis was tested anatomically, taking advantage of the availability of a large variety of neuroanatomical tracers injected simultaneously in distinct cortical areas in the same animal (Cappe et al., 2009a). We performed in two macaque monkeys injections of neuroanatomical tracers in the rostral (RAC) and caudal auditory cortex (CAC), posterior parietal somatosensory cortex 
(PE and PEa in area 5), and dorsal and ventral premotor cortical areas (PMd, PMv) in order to assess their connectivity with the thalamus (Cappe et al., 2009a). The results demonstrate the existence of thalamic nuclei exhibiting territories of projection to the cerebral cortex overlapping across different modalities (sensory and motor; Fig. 2). Indeed, thalamocortical projections, distinct from the ones arising from specific unisensory sensory nuclei, were observed from motor thalamic nuclei, such as the ventroanterior nucleus (VA) to PE and to PEa. The central lateral nucleus (CL) and the mediodorsal nucleus (MD) project to RAC, CAC, PEa, PE, PMd and PMv. We found common territories of projection from the nucleus LP to PMd, PMv, PEa and PE. It is also is of interest to note that the medial geniculate nucleus (MGN), known as the main auditory thalamic relay, projects also to the parietal cortex (PE in particular) and the premotor cortex (PMd/PMv). Moreover, the thalamic nucleus exhibiting the most significant overlap across modalities is the medial pulvinar nucleus (PuM) with projections from superimposed territories to all six cortical areas injected with tracers. Projections from PuM to the auditory cortex were also described by de la Mothe and her colleagues (2006b). We also found projections to the areas injected from thalamic nuclei that are more commonly considered as multisensory, such as the suprageniculate nucleus and the limitans nucleus. All these results are consistent with the presence of thalamic territories possibly integrating different sensory modalities with motor attributes. In addition, considering also in this context the corticothalamic projection systems, the thalamus could represent an alternative path-

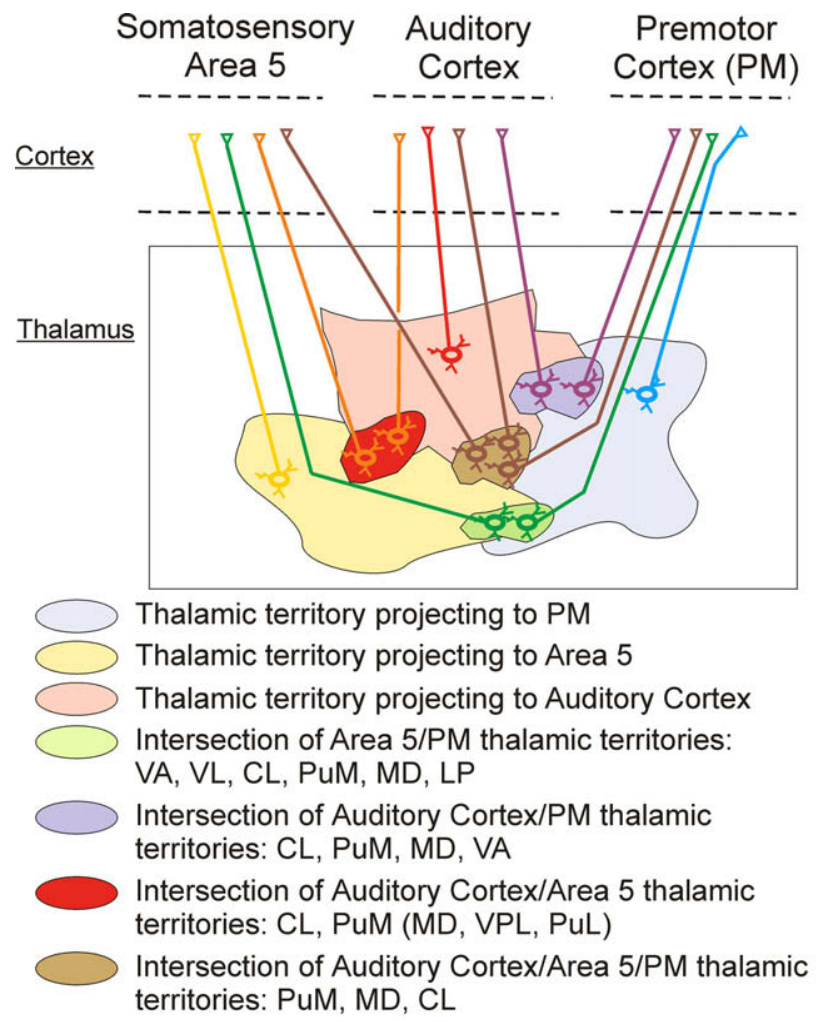

Cortico-thalamic projections from Area 5 to thalamic nuclei: PuM, LP, VPL, CM, CL, MD

Fig. 2. Thalamo-cortical (TC) connections originating from different thalamic nuclei to distinct sensory (somatosensory area 5 - areas PE and PEa - and auditory areas mainly the belt and the parabelt of the auditory cortex) and motor cortical areas (modified from Cappe et al., 2009a). Thalamic nuclei where overlaps of TC connections occur are mentioned below the diagram. Thalamic nuclei which contain cortico-thalamic connections (giant and small endings) from area 5 are also indicated below the diagram. way to direct corticocortical interactions, by which information can be transferred trans-thalamically across remote cortical areas belonging to different sensory and/or motor modalities (Fig. 2).

\subsection{Overlap between thalamocortical (TC) connections in the thalamus and corticothalamic (CT) connections}

The calculation of the degree of overlap throughout the thalamus between the different TC connections (Tanné-Gariépy et al., 2002; Morel et al., 2005; Cappe et al., 2009a) allowed us to determine that the projections to areas of a same modality, such premotor areas have a degree of overlap of $35 \%$ on average. If we consider projections to areas of different modalities, there is a degree of overlap that ranges from $4 \%$ to $12 \%$ throughout the thalamus between the auditory and premotor cortical areas and it can reach $30 \%$ in the caudal part of the thalamus. There is therefore a most predominant degree of divergence in this part of the thalamus. In addition, we conducted the same calculation of degree of overlap focusing on the PuM nucleus because it appeared to be the thalamic nucleus where the maximum overlap between TC projections was observed. The TC afferents to auditory and premotor areas overlap in PuM to a degree ranging from $14 \%$ to $20 \%$.

The significant overlap between the projections to the auditory and premotor cortex in the thalamus is located mainly in the PuM, VA and CL nuclei. The overlap between the projections to auditory and parietal cortical areas concerns different thalamic nuclei such as PuM, CL and, to a lesser extent, LP and PuL. The projections to the premotor and posterior parietal cortex overlap primarily in PuM, LP, MD and also in VA, VLpd and CL. At the PuM level, the entanglement between projections to the different injected cortical areas ranges from $2 \%$ to $23 \%$ across the cortical areas. In the PuM, the main overlap is for projections directed to the auditory cortex and premotor cortex. The notion that some thalamic nuclei can contain an overlap between thalamocortical connections to different sensory and premotor areas is illustrated in Fig. 2 (CT connections from area 5 are also indicated). Furthermore, we observed that the thalamic nuclei PuM, LP and, to a lesser extent, VPL, CM, $\mathrm{CL}$ and $\mathrm{MD}$ contained both the terminal fields of $\mathrm{CT}$ projections from parietal areas PE and PEa (PEa only for MD), and the neurons of origin of TC projections to auditory, parietal and premotor areas. These results show that the connectivity of some nuclei in the thalamus (PuM, LP, VPL, CM, CL and MD) could enable them to integrate information from different sensory modalities with or without motor attributes (Cappe et al., 2007a, 2009a). Indeed, these nuclei receive projections from parietal areas (Cappe et al., 2007a) and send projections to premotor and auditory areas. These interactions between the thalamus and the cortex could play a role in multisensory and sensorimotor integration.

\subsection{Role of the pulvinar}

As previously described, the premotor cortex is an area of multisensory integration because it is a zone of convergence of visual, auditory and somatosensory inputs (Graziano et al., 2000; Graziano, 2001). Sensory responses recorded in the premotor cortex in monkeys are related to the motor intention, implying a role in the preparation of voluntary movements (Boussaoud et al., 1995; Wise et al., 1997). These multimodal inputs to the premotor cortex come both from sensory association areas, and also from multisensory areas of the parietal lobe (Tanné-Gariépy et al., 2002; Johnson et al., 1996; Luppino et al. 1999; Shipp et al., 1998). The latencies of the responses to sensory stimuli in the premotor cortex may be relatively short, which is compatible with direct access through the thalamus, for example, in agreement with recent anatomical studies (Morel et al., 2005; Cappe et al., 2009a) showing the existence of inputs from PuM directed towards PMd and PMv. Indeed, the 
medial part of the pulvinar nucleus is the main candidate (although other thalamic nuclei such as LP, VPL, MD or CL may also play a role) to represent an alternative to cortico-cortical loops by which information can be transferred between cortical areas belonging to different sensory and sensorimotor modalities (Shipp, 2003). Interestingly, PuM also receives direct inputs from the superior colliculus (Stepniewska, 2004); information which is already multimodal. It could be that loops between the cerebral cortex and the pulvinar nucleus are part of mechanisms involved in multisensory integration observed in unisensory cortical areas (Crick and Koch, 1998).

\section{Role of non-specific connections?}

Previous psychophysical studies in humans have shown that a multisensory stimulation can give rise to a percept that differs from that derived from a single modality (e.g. Stein and Meredith, 1993). Such multisensory processing can affect a range of different behavioral parameters, such as reaction times (Welch and Warren, 1986; Raab, 1962), stimulus detection rate (Grant and Seitz, 2000), accuracy of stimulus identification (Giard and Peronnet, 1999) as well as learning effects on stimulus processing (Murray et al., 2005; Lehmann and Murray, 2005). While for example the decrease in reaction times under multisensory conditions have been largely reported for human subjects in auditory-visual recognition tasks, no behavioral data to our knowledge are available in monkeys performing similar protocols. This lack of evidence in the behaving monkey is regrettable as most of the heteromodal connections have been revealed in non-human primates (see above). Indeed, to investigate the role of these heteromodal connections, we first searched for evidence of multisensory integration in the monkey at the behavioral level, and then we investigated their functional impact at the single cell level.

\subsection{Behavioral evidence in the monkey}

In a recent experiment, monkeys were trained to perform a simple detection task with visual, auditory or audio-visual stimuli (Cappe et al., 2007b). The stimuli were presented with different intensities and the reaction times (RTs) to detect theses stimuli were compared. By varying the intensity of the individual auditory and visual stimuli we have observed that under near-threshold conditions, the multisensory stimuli had a significant facilitative effect on reaction times and stimulus detection, a phenomenon named the "redundant signal effect" (RSE; Raab, 1962). Two classes of models can explain this effect: race and co-activation models. According to the race model (Raab, 1962), neural interactions are not required to obtain the RSE. Rather, stimuli independently compete for response initiation and the faster of the two mediates behavior on any given trial. Thus, simple probability summation could produce the RSE. In contrast to this model, we observed in the monkey shorter RTs for the multisensory stimuli than those obtained for each unisensory stimuli, suggesting that the two components of a multisensory stimulus are not processed independently, but rather converge and are integrated in the central nervous system. These data are in line with co-activation models (e.g., Miller, 1982), in which neural responses from stimulus pairs interact and are pooled prior to behavioral response initiation, for which the threshold is met more rapidly by multisensory stimuli than by unisensory stimuli. In the monkey we have shown that the auditory-visual effect, violating the "Race Model" (Miller, 1982), disappeared at high intensities inferring that multisensory interaction in the behaving monkey obeys the rule of inverse effectiveness that proposes a higher multisensory benefit when the unisensory stimuli are weak (Cappe et al., 2007b). The main unanswered ques- tion is where in the brain is computed this integration that leads to an increased efficiency of the sensory-motor output. However, recent evidence points to a link between early non-linear auditorysomatosensory interactions within posterior auditory cortices with faster vs. slower RTs (Sperdin et al., 2009).

\subsection{Electrophysiological evidence in the primary sensory areas}

As mentioned earlier (see also other chapters in this issue), many human studies conducted with EEG or fMRI have revealed multisensory interactions in low-level cortices such as V1 (see Ghazanfar and Schroeder, 2006 for a review and Martuzzi et al., 2007; Romei et al., 2007, 2008 for recent evidences) and/or at a latency that strongly suggests that such phenomena are supported by the heteromodal connections directly linking areas of different sensory modalities. For example, the visual information derived from the lip movements during speech processing can affect directly the responses recorded in the auditory cortex (Besle et al., 2004).

In the non-human primate (see also other chapters in this issue), several electrophysiological experiments have similarly revealed audio-visual interactions in the auditory cortex (Ghazanfar et al., 2005; Bizley et al., 2007; Bizley and King, 2008; Cappe et al., 2007b; Kayser et al., 2008) down to the level of A1 (Kayser et al., 2008). These recent results were highly complementary to the pioneer works of Schroeder's laboratory that have revealed the multimodal feature of the monkey auditory belt where both visual and somatosensory responses can be evoked (Schroeder et al., 2001; Schroeder and Foxe, 2002; Fu et al., 2003). In the carnivore, while previous studies have reported that the visual cortex can be activated by auditory stimuli (Spinelli et al., 1968; Morrell, 1972; Fishman and Michael, 1973), intracellular recording in the primary visual cortex (A17) have recently failed to find such auditory responses (Sanchez-Vives et al., 2006). Consequently, because no evidence of auditory or visuo-auditory activity was reported at the single cell level in the awake monkey and to investigate the role of the $\mathrm{A} 1$ to $\mathrm{V} 1$ projection described in the macaque, we performed an electrophysiological study of the effect of an auditory stimulus on the neuronal activity of area V1 in a behaving monkey performing an oculomotor task (Wang et al., 2008). Three main points have to be highlighted from this study. First, we did not find any auditory response in the set of single units we tested. This is similar to what was reported in the primary auditory cortex as electrophysiological recordings suggest that non-auditory events are rather of modulatory influence and do not drive activation at the spiking level, but rather are more evident in post-synaptic potentials (Lakatos et al., 2007). Second, in the superior colliculus or in higher-order multisensory cortical areas of the monkey such as the prefrontal, parietal or inferotemporal areas, the convergence of different sensory modalities is reflected mainly by a modulation (enhancement or depression) of the strength of the sensory responses according to the spatial and temporal congruencies of the two stimuli (Stein, 1998; Avillac et al., 2007; Romanski, 2007). In V1 when presenting a spatially congruent sound, we did not observe an enhancement in visual neuronal activity even at low visual contrast. However, the visual latency of V1 neurons was reduced by about 5\% during a visuoauditory stimulation when using low saliency visual stimuli. The superior colliculus, which is directly involved in the control of oculomotor behavior (Sparks, 2002), receives about a third of its cortical afferents from the primary visual cortex (Collins et al., 2005). Consequently, the decrease in V1 response latencies during multisensory stimulation can act directly on the response of cells in the SC and speed up the initiation of the saccadic command by the brain stem oculomotor nucleus as demonstrated in the behaving monkey (Bell et al., 2005). Lastly, the visuo-auditory interactions 
are dependent of the perceptual task in which the animal was engaged. During a passive fixation task, V1 responses are not affected by the simultaneous presentation a sound. The effect on responses latencies is only observed when the visual cue is the target of the ocular saccade. These results are in complete agreement with human studies showing different patterns of multisensory integration according to the behavioral context. First, in humans, the detection or discrimination of multisensory objects, as well as the perceptual expertise of subjects, differentially affect the temporal aspects at which multisensory interactions occur (Fort et al., 2002). Second, in blind patients, the cross-modal compensation that occurs following sensory deprivation (Bavelier and Neville, 2002; Bavelier et al., 2006) is expressed as a colonization of the visual areas by somatosensory processing during Braille reading but mainly during an active discrimination task (Sadato and Hallett, 1999; see also Amedi et al., 2007).

To summarize, multisensory interactions can be expressed differently as a function of the specific cortical areas under investigation. In primary areas such as V1 and A1, multisensory interaction is predominantly of modulatory influence with no (or weak) response to non-specific sensory stimuli. On the other hand, in association areas multisensory stimuli have a more pronounced impact on both the strength and latencies of neuronal responses with a proportion of cells showing such modulation being largely variable across these areas (see Avillac et al., 2007; Romanski, 2007; Bizley et al., 2007).

\section{Conclusion}

Thus, recent anatomical and electrophysiological approaches provide evidence that multisensory interactions can be observed at early stages of sensory processing. However, the complexity of the connectivity network involved in multisensory interplay, that includes cortical and thalamo-cortical pathways as well as the diversity of interactions observed across the thalamus, the cortical sensory or associative areas, favor a distributed system that is probably adapted to specific behavioral situations. This is suggested by the fact that heteromodal connections that have thus far been identified are organized topologically and that at the neuronal level, in multisensory situations the perceptual load of the task will likely affect differently the neuronal responses. For this reason, multisensory interactions are probably specific to each individual area and only a comparative study of how neuronal properties are diversified across areas would provide a better comprehension of multisensory interplay.

\section{Acknowledgements}

We thank M. Murray for correcting and comments on the text. Grants supports: the CNRS ATIP program (to P.B.), the Swiss National Science Foundation, grants 31-61857.00 (to E.M.R.) and 310000-110005 (to E.M.R.), the Swiss National Science Foundation Center of Competence in Research (NCCR) on "Neural plasticity and Repair » (to E.M.R.).

\section{References}

Amedi, A., Jacobson, G., Hendler, T., Malach, R., Zohary, E., 2002. Convergence of visual and tactile shape processing in the human lateral occipital complex. Cereb. Cortex 12, 1202-1212.

Amedi, A., Stern, W.M., Camprodon, J.A., Bermpohl, F., Merabet, L., Rotman, S., Hemond, C., Meijer, P., Pascual-Leone, A., 2007. Shape conveyed by visual-toauditory sensory substitution activates the lateral occipital complex. Nat. Neurosci. 10, 687-689.

Andersen, R.A. Asanuma, C. Essick, G. Siegel, R.M. 1990. Corticocortical connections of anatomically and physiologically defined subdivisions within the inferior parietal lobule. J. Comp. Neurol. 296, 65-113.
Andersen, R.A., Snyder, L.H., Bradley, D.C., Xing, J., 1997. Multimodal representation of space in the posterior parietal cortex and its use in planning movements. Annu. Rev. Neurosci. 20, 303-330.

Avillac, M., Ben Hamed, S., Duhamel, J.R., 2007. Multisensory integration in the ventral intraparietal area of the macaque monkey. J. Neurosci. 27, 1922-1932.

Barbas, H., Rempel-Clower, N., 1997. Cortical structure predicts the pattern of corticocortical connections. Cereb. Cortex 7, 635-646.

Barone, P., Clarey, J.C., Irons, W.A., Imig, T.J., 1996. Cortical synthesis of azimuthsensitive single-unit responses with nonmonotonic level tuning: a thalamocortical comparison in the cat. J. Neurophysiol. 75 (3), 1206-1220.

Barth, D.S., Goldberg, N., Brett, B., Di, S., 1995. The spatiotemporal organization of auditory, visual, and auditory-visual evoked potentials in rat cortex. Brain Res. $678,177-190$.

Batardiere, A., Barone, P., Dehay, C., Kennedy, H., 1998. Area- specific laminar distribution of cortical feedback neurons projecting to cat area 17: quantitative analysis in the adult and during ontogeny. J. Comp. Neurol. 396, 493-510.

Bavelier, D., Neville, H.J., 2002. Cross-modal plasticity: where and how? Nat. Rev. Neurosci. 3 (6), 443-452.

Bavelier, D., Dye, M.W., Hauser, P.C., 2006. Do deaf individuals see better? Trends Cogn. Sci. 10 (11), 512-518.

Baylis, G.C., Rolls, E.T., Leonard, C.M., 1987. Functional subdivisions of the temporal lobe neocortex. J. Neurosci. 7, 330-342.

Bell, A.H., Meredith, M.A., Van Opstal, A.J., Munoz, D.P., 2005. Crossmodal integration in the primate superior colliculus underlying the preparation and initiation of saccadic eye movements. J. Neurophysiol. 93, 3659-3673.

Besle, J., Fort, A., Delpuech, C., Giard, M.H., 2004. Bimodal speech: early suppressive visual effects in human auditory cortex. Eur. J. Neurosci. 20, 2225-2234.

Bizley, J.K., Nodal, F.R., Bajo, V.M., Nelken, I., King, A.J., 2007. Physiological and anatomical evidence for multisensory interactions in auditory cortex. Cereb. Cortex 17, 2172-2189.

Bizley, J.K., King, A.J., 2008. Visual-auditory spatial processing in auditory cortical neurons. Brain Res. 1242, 24-36.

Blatt, G.J., Andersen, R.A., Stoner, G.R., 1990. Visual receptive field organization and cortico-cortical connections of the lateral intraparietal area (area LIP) in the macaque. J. Comp. Neurol. 299, 421-445.

Boussaoud, D., di Pellegrino, G., Wise, S.P., 1995. Frontal lobe mechanisms subserving vision-for- action versus vision-for-perception. Behav. Brain Res. $72,1-15$.

Bremmer, F., Klam, F., Duhamel, J.R., Ben Hamed, S., Graf, W., 2002. Visual-vestibular interactive responses in the macaque ventral intraparietal area (VIP). Eur. J. Neurosci. 16, 1569-1586.

Bruce, C., Desimone, R., Gross, C.G., 1981. Visual properties of neurons in a polysensory area in superior temporal sulcus of the macaque. J. Neurophysiol. 46, 369-384.

Buchel, C., Price, C., Friston, K.A., 1998. A multimodal language region in the ventral visual pathway. Nature 394, 274-277.

Budinger, E., Heil, P., Hess, A., Scheich, H., 2006. Multisensory processing via early cortical stages: connections of the primary auditory cortical field with other sensory systems. Neuroscience 143, 1065-1083.

Bullier, J., 2006. In: Leo van Hemmen, J., Sejnowslci, Terrence J. (Eds.), What is Feed Back ? 23 Problems in Systems Neuroscience.

Calvert, G.A., Brammer, M.J., Bullmore, E.T., Campbell, R., Iversen, S.D., David, A.S., 1999. Response amplification in sensory-specific cortices during crossmodal binding. Neuroreport 10, 2619-2623.

Calvert, G.A., Hansen, P.C., Iversen, S.D., Brammer, M.J., 2001. Detection of audiovisual integration sites in humans by application of electrophysiological criteria to the BOLD effect. Neuroimage 14, 427-438.

Cappe, C., Barone, P., 2005. Heteromodal connections supporting multisensory integration at low levels of cortical processing in the monkey. Eur. J. Neurosci. 22, 2886-2902.

Cappe, C., Morel, A., Rouiller, E.M., 2007a. Thalamocortical and the dual pattern of corticothalamic projections of the posterior parietal cortex in macaque monkeys. Neuroscience 146, 1371-1387.

Cappe, C., Loquet, G., Barone, P., Rouiller, E.M., 2007b. Influences of auditory and visual stimuli on neurons in auditory cortical areas in monkeys performing an audio-visual detection task. In: Society for Neuroscience Meeting, San Diego (USA), abstr. 620.18-SS4.

Cappe, C., Morel, A., Barone, P., Rouiller, E.M., 2009a. The thalamocortical projection systems in primate: an anatomical support for multisensory and sensorimotor integrations. Cereb. Cortex. doi:10.1093/cercor/bhn228.

Cappe, C., Thut, G., Romei, V., Murray, M.M., 2009b. Selective integration of auditory-visual looming cues by humans. Neuropsychologia 47, 1045-1052.

Clarey, J.C., Barone, P., Irons, W.A., Samson, F.K., Imig, T.J., 1995. Comparison of noise and tone azimuth tuning of neurons in cat primary auditory cortex and medical geniculate body. J. Neurophysiol. 74 (3), 961-980.

Cohen, Y.E., Russ, B.E., Gifford 3rd., G.W., 2005. Auditory processing in the posterior parietal cortex. Behav. Cogn. Neurosci. Rev. 4, 218-231. Review.

Collins, C.E., Lyon, D.C., Kaas, J.H., 2005. Distribution across cortical areas of neurons projecting to the superior colliculus in new world monkeys. Anat. Rec. A. Discov. Mol. Cell. Evol. Biol. 285, 619-627.

Cooke, D.F., Graziano, M.S., 2003. Defensive movements evoked by air puff in monkeys. J. Neurophysiol. 90, 3317-3329.

Cooke, D.F., Graziano, M.S., 2004. Sensorimotor integration in the precentral gyrus: polysensory neurons and defensive movements. J. Neurophysiol. 91,1648 1660. 
Crick, F., Koch, C., 1998. Constraints on cortical and thalamic projections: the nostrong-loops hypothesis. Nature 391, 245-250.

Darian-Smith, C., Tan, A., Edwards, S., 1999. Comparing thalamocortical and corticothalamic microstructure and spatial reciprocity in the macaque ventral posterolateral nucleus (VPLc) and medial pulvinar. J. Comp. Neurol. 410, 211234.

de la Mothe, L.A., Blumell, S., Kajikawa, Y., Hackett, T.A., 2006a. Cortical connections of the auditory cortex in marmoset monkeys: core and medial belt regions. J. Comp. Neurol. 496, 27-71.

de la Mothe, L.A., Blumell, S., Kajikawa, Y., Hackett, T.A., 2006b. Thalamic connections of the auditory cortex in marmoset monkeys: core and medial belt regions. J. Comp. Neurol. 496, 72-96.

Driver, J., Noesselt, T., 2008. Multisensory interplay reveals crossmodal influences on 'sensory-specific' brain regions, neural responses, and judgments. Neuron $57,11-23$.

Duhamel, J.R., Colby, C.L., Goldberg, M.E., 1998. Ventral intraparietal area of the macaque: congruent visual and somatic response properties. J. Neurophysiol. 79, 126-136.

Falchier, A., Clavagnier, S., Barone, P., Kennedy, H., 2002. Anatomical evidence of multimodal integration in primate striate cortex. J. Neurosci. 22, 5749-5759.

Feig, S., Harting, J.K., 1998. Corticocortical communication via the thalamus: Ultrastructural studies of corticothalamic projections from area 17 to the lateral posterior nucleus of the cat and inferior pulvinar nucleus of the owl monkey. J. Comp. Neurol. 395, 281-295.

Felleman, D.J., Van Essen, D.C., 1991. Distributed hierarchical processing in the primate cerebral cortex. Cereb. Cortex 1, 1-47.

Fishman, M.C., Michael, P., 1973. Integration of auditory information in the cat's visual cortex. Vision Res. 13, 1415-1419.

Fort, A., Delpuech, C., Pernier, J., Giard, M.H., 2002. Dynamics of corticosubcortical cross-modal operations involved in audio-visual object detection in humans. Cereb. Cortex 12, 1031-1039.

Foxe, J.J., Morocz, I.A., Murray, M.M., Higgins, B.A., Javitt, D.C., Schroeder, C.E., 2000. Multisensory auditory-somatosensory interactions in early cortical processing revealed by high-density electrical mapping. Brain Res. Cogn. Brain Res. 10, 7783.

Fu, K.M., Johnston, T.A., Shah, A.S., Arnold, L., Smiley, J., Hackett, T.A., Garraghty, P.E., Schroeder, C.E., 2003. Auditory cortical neurons respond to somatosensory stimulation. J Neurosci. 23, 7510-7515.

Fuster, J.M., Bodner, M., Kroger, J.K., 2000. Cross-modal and cross-temporal association in neurons of frontal cortex. Nature 405, 347-351.

Gaffan, D., Harrison, S., 1991. Auditory-visual associations, hemispheric specialization and temporal-frontal interaction in the rhesus monkey. Brain $114,2133-2144$

Galletti, C., Gamberini, M., Kutz, D.F., Fattori, P., Luppino, G., Matelli, M., 2001. The cortical connections of area V6: an occipito-parietal network processing visual information. Eur. J. Neurosci. 13, 1572-1588.

Gattass, R., Oswaldo-Cruz, E., Sousa, A.P., 1979. Visual receptive fields of units in the pulvinar of cebus monkey. Brain Res. 160, 413-430.

Ghazanfar, A.A., Maier, J.X., Hoffman, K.L., Logothetis, N.K., 2005. Multisensory integration of dynamic faces and voices in rhesus monkey auditory cortex. J. Neurosci. 25, 5004-5012.

Ghazanfar, A.A., Schroeder, C.E., 2006. Is neocortex essentially multisensory? Trends Cogn. Sci. 10, 278-285. Review.

Giard, M.H., Peronnet, F., 1999. Auditory-visual integration during multimodal object recognition in humans: a behavioral and electrophysiological study. J. Cogn. Neurosci. 11, 473-490.

Gonchar, Y., Burkhalter, A., 1999. Differential subcellular localization of forward and feedback interareal inputs to parvalbumin expressing GABAergic neurons in rat visual cortex. J. Comp. Neurol. 406, 346-360.

Gottlieb, J., 2007. From thought to action: the parietal cortex as a bridge between perception, action, and cognition. Neuron 53, 9-16. Review.

Grant, K.W., Seitz, P.F., 2000. The use of visible speech cues for improving auditory detection of spoken sentences. J. Acoust. Soc. Am. 108 (3 Pt 1), 1197-1208.

Graziano, M.S.A., Gross, C.G., 1998. Spatial maps for the control of movement. Curr. Opin. Neurobiol. 8, 195-201.

Graziano, M.S., Cooke, D.F., Taylor, C.S., 2000. Coding the location of the arm by sight. Science 290, 1782-1786.

Graziano, M.S., 2001. A system of multimodal areas in the primate brain. Neuron 29, 4-6.

Gutierrez, C., Cola, M.G., Seltzer, B., Cusick, C., 2000. Neurochemical and connectional organization of the dorsal pulvinar complex in monkeys. J. Comp. Neurol. 419, 61-86.

Hackett, T.A., Stepniewska, I., Kaas, J.H., 1998. Thalamocortical connections of the parabelt auditory cortex in macaque monkeys. J. Comp. Neurol. 400, 271-286.

Hall, A.J., Lomber, S.G., 2008. Auditory cortex projections target the peripheral field representation of primary visual cortex. Exp. Brain Res. 190, 413-430.

Heffner, R.S., Heffner, H.E., 1992. Visual factors in sound localization in mammals. J. Comp Neurol. 317, 219-232.

Hikosaka, K., Iwai, E., Saito, H., Tanaka, K., 1988. Polysensory properties of neurons in the anterior bank of the caudal superior temporal sulcus of the macaque monkey. J. Neurophysiol. 60, 1615-1637.

Huffman, K.J., Krubitzer, L., 2001. Area 3a: topographic organization and cortical connections in marmoset monkeys. Cereb. Cortex 11, 849-867.

Hupé, J.M., James, A.C., Payne, B.R., Lomber, S.G., Girard, P., Bullier, J., 1998. Cortical feedback improves discrimination between figure and background by V1, V2 and V3 neurons. Nature 394, 784-787.
Innocenti, G.M., Berbel, P., Clarke, S., 1988. Development of projections from auditory to visual areas in the cat. J. Comp. Neurol. 272, 242-259.

Johnson, P.B., Ferraina, S., Bianchi, L., Caminiti, R., 1996. Cortical networks for visual reaching: physiological and anatomical organization of frontal and parietal lobe arm regions. Cereb. Cortex 6, 102-119.

Jones, E.G., Powell, T.P., 1970. An anatomical study of converging sensory pathways within the cerebral cortex of the monkey. Brain 93, 793-820.

Kaas, J.H., Morel, A., 1993. Connections of visual areas of the upper temporal lobe of owl monkeys: the MT crescent and dorsal and ventral subdivisions of FST. J. Neurosci. 13, 534-546.

Kayser, C., Petkov, C.I., Augath, M., Logothetis, N.K., 2007. Functional imaging reveals visual modulation of specific fields in auditory cortex. J. Neurosci. 27, 18241835.

Kayser, C., Petkov, C.I., Logothetis, N.K., 2008. Visual modulation of neurons in auditory cortex. Cereb. Cortex 18, 1560-1574.

Kriegstein, K.V., Kleinschmidt, A., Sterzer, P., Giraud, A.L., 2005. Interaction of face and voice areas during speaker recognition. J. Cogn. Neurosci. 17, 367-376.

Krubitzer, L.A., Kaas, J.H., 1990. The organization and connections of somatosensory cortex in marmosets. J. Neurosci. 10, 952-974.

Lakatos, P., Chen, C.M., O'Connell, M.N., Mills, A., Schroeder, C.E., 2007. Neuronal oscillations and multisensory interaction in primary auditory cortex. Neuron 53, 279-292.

Lehmann, S., Murray, M.M., 2005. The role of multisensory memories in unisensory object discrimination. Brain. Res. Cogn. Brain Res. 24, 326-334.

Lewis, J.W., Van Essen, D.C., 2000. Corticocortical connections of visual, sensorimotor, and multimodal processing areas in the parietal lobe of the macaque monkey. J. Comp. Neurol. 428, 112-137.

Luppino, G., Murata, A., Govoni, P., Matelli, M., 1999. Largely segregated parietofrontal connections linking rostral intraparietal cortex (areas AIP and VIP) and the ventral premotor cortex (areas F5 and F4). Exp. Brain Res. 128, 181-187.

Lyon, D.C., Kaas, J.H., 2001. Connectional and architectonic evidence for dorsal and ventral V3, and dorsomedial area in marmoset monkeys. J. Neurosci. 21, 249261.

Macaluso, E., Frith, C.D., Driver, J., 2000. Modulation of human visual cortex by crossmodal spatial attention. Science 289, 1206-1208.

Maier, J.X., Neuhoff, J.G., Logothetis, N.K., Ghazanfar, A.A., 2004. Multisensory integration of looming signals by rhesus monkeys. Neuron 43, 177-181.

Maier, J.X., Chandrasekaran, C., Ghazanfar, A.A., 2008. Integration of bimodal looming signals through neuronal coherence in the temporal lobe. Curr. Biol. 18, 963-968.

Martuzzi, R., Murray, M.M., Michel, C.M., Thiran, J.P., Maeder, P.P., Clarke, S., Meuli, R.A., 2007. Multisensory interactions within human primary cortices revealed by BOLD dynamics. Cereb. Cortex 17, 1672-1679.

Maunsell, J.H., Van Essen, D.C., 1983. The connections of the middle temporal visual area (MT) and their relationship to a cortical hierarchy in the macaque monkey. J. Neurosci. 3, 2563-2586.

Miller, J., 1982. Divided attention: evidence for coactivation with redundant signals. Cognit. Psychol. 14, 247-279.

Miller, L.M., D’Esposito, M., 2005. Perceptual fusion and stimulus coincidence in the cross-modal integration of speech. J. Neurosci. 25, 5884-5893.

Miller, M.W., Vogt, B.A., 1984. Direct connections of rat visual cortex with sensory, motor, and association cortices. J. Comp. Neurol. 226, 184-202.

Molholm, S., Ritter, W., Murray, M.M., Javitt, D.C., Schroeder, C.E., Foxe, J.J., 2002. Multisensory auditory-visual interactions during early sensory processing in humans: a high-density electrical mapping study. Brain Res. Cogn. Brain Res. 14, 115-128.

Morel, A., Liu, J., Wannier, T., Jeanmonod, D., Rouiller, E.M., 2005. Divergence and convergence of thalamocortical projections to premotor and supplementary motor cortex: a multiple tracing study in macaque monkey. Eur. J. Neurosci. 21, 1007-1029.

Morrell, F., 1972. Visual system's view of acoustic space. Nature 238, 44-46.

Murray, M.M., Molholm, S., Michel, C.M., Heslenfeld, D.J., Ritter, W., Javitt, D.C., Schroeder, C.E., Foxe, J.J., 2005. Grabbing your ear: rapid auditorysomatosensory multisensory interactions in low-level sensory cortices are not constrained by stimulus alignment. Cereb. Cortex 15, 963-974.

Nascimento-Silva, S., Schroeder, C.E., Hackett, T.A., Ulbert, I., Karmos, G., Smiley, J.F., 2005. Projections from visual area V2 to auditory area Tpt in the superior temporal gyrus. In: Society for Neuroscience Meeting, Washington (USA), abstr. 617.16.

Ojima, H., 1994. Terminal morphology and distribution of corticothalamic fibers originating from layers 5 and 6 of cat primary auditory cortex. Cereb. Cortex 4, 646-663.

Palmer, S.M., Rosa, M.G., 2006. A distinct anatomical network of cortical areas for analysis of motion in far peripheral vision. Eur. J. Neurosci. 24, 2389-2405.

Pandya, D.N., Seltzer, B., 1982. Intrinsic connections and architectonics of posterior parietal cortex in the rhesus monkey. J. Comp. Neurol. 204, 196-210.

Raab, D.H., 1962. Statistical facilitation of simple reaction times. Trans. NY Acad. Sci. 24, 574-590.

Rempel-Clower, N.L., Barbas, H., 2000. The laminar pattern of connections between prefrontal and anterior temporal cortices in the Rhesus monkey is related to cortical structure and function. Cereb. Cortex 10, 851-865.

Rockland, K.S., 1996. Two types of corticopulvinar terminations: round (type 2) and elongate (type 1). J. Comp. Neurol. 368, 57-87.

Rockland, K.S., Ojima, H., 2003. Multisensory convergence in calcarine visual areas in macaque monkey. Int. J. Psychophysiol. 50, 19-26. 
Romanski, L.M., Giguere, M., Bates, J.F., Goldman-Rakic, P.S., 1997. Topographic organization of medial pulvinar connections with the prefrontal cortex in the rhesus monkey. J. Comp. Neurol. 379, 313-332.

Romanski, L.M., Bates, J.F., Goldman-Rakic, P.S., 1999. Auditory belt and parabelt projections to the prefrontal cortex in the rhesus monkey. J. Comp. Neurol. 403, 141-157.

Romanski, L.M., 2007. Representation and integration of auditory and visual stimuli in the primate ventral lateral prefrontal cortex. Cereb. Cortex 17 (Suppl. 1), i6169.

Romei, V., Murray, M.M., Merabet, L.B., Thut, G., 2007. Occipital transcranial magnetic stimulation has opposing effects on visual and auditory stimulus detection: implications for multisensory interactions. J. Neurosci. 27, 1146511472.

Romei, V., Murray, M.M., Cappe, C., Thut, G., 2008. Looming sounds selectively enhance visual excitability. 9th International Multisensory Research Forum, Hamburg.

Rouiller, E.M., Tanné, J., Moret, V., Kermadi, I., Boussaoud, D., Welker, E., 1998. Dual morphology and topography of the corticothalamic terminals originating from the primary, supplementary motor, and dorsal premotor cortical areas in macaque monkeys. J. Comp. Neurol. 396, 169-185.

Rouiller, E.M., Welker, E., 2000. A comparative analysis of the morphology of corticothalamic projections in mammals. Brain Res. Bull. 53, 727-741.

Rouiller, E.M., Wannier, T., Morel, A., 2003. The dual pattern of corticothalamic projection of the premotor cortex in macaque monkeys. Thalamus Relat. Syst. 2, 189-197.

Rouiller, E.M., Durif, C., 2004. The dual pattern of corticothalamic projection of the primary auditory cortex in macaque monkey. Neurosci. Lett. 358, 49-52.

Russ, B.E., Kim, A.M., Abrahamsen, K.L., Kiringoda, R., Cohen, Y.E., 2006. Responses of neurons in the lateral intraparietal area to central visual cues. Exp. Brain Res. $174,712-727$.

Sadato, N., Hallett, M., 1999. FMRI occipital activation by tactile stimulation in a blind man. Neurology 52 (2), 423.

Salin, P.A., Bullier, J., 1995. Corticocortical connections in the visual system: structure and function. Physiol. Rev. 75, 107-154.

Sanchez-Vives, M.V., Nowak, L.G., Descalzo, V.F., Garcia-Velasco, J.V., Gallego, R., Berbel, P., 2006. Crossmodal audio-visual interactions in the primary visual cortex of the visually deprived cat: a physiological and anatomical study. Prog. Brain Res. 155, 287-311.

Schall, J.D., Morel, A., King, D.J., Bullier, J., 1995. Topography of visual cortex connections with frontal eye field in macaque: convergence and segregation of processing streams. J. Neurosci. 15, 4464-4487.

Schlack, A., Sterbing-D’Angelo, S.J., Hartung, K., Hoffmann, K.P., Bremmer, F., 2005. Multisensory space representations in the macaque ventral intraparietal area. J. Neurosci. 25, 4616-4625.

Schroeder, C.E., Lindsley, R.W., Specht, C., Marcovici, A., Smiley, J.F., Javitt, D.C., 2001. Somatosensory input to auditory association cortex in the macaque monkey. J. Neurophysiol. 85, 1322-1327.

Schroeder, C.E., Foxe, J.J., 2002. The timing and laminar profile of converging inputs to multisensory areas of the macaque neocortex. Cognit. Brain Res. 14, 187-198.

Schwartz, M.L., Dekker, J.J., Goldman-Rakic, P.S., 1991. Dual mode of corticothalamic synaptic termination in the mediodorsal nucleus of the Rhesus monkey. J. Comp. Neurol. 309, 289-304.

Seltzer, B., Pandya, D.N., 1994. Parietal, temporal, and occipital projections to cortex of the superior temporal sulcus in the rhesus monkey: a retrograde tracer study. J. Comp. Neurol. 343, 445-463.

Senkowski, D., Talsma, D., Grigutsch, M., Herrmann, C.S., Woldorff, M.G., 2007. Good times for multisensory integration: effects of the precision of temporal synchrony as revealed by gammaband oscillations. Neuropsychologia 45 , 561-571.

Sherman, S.M., Guillery, R.W., 2002. The role of the thalamus in the flow of information to the cortex. Philos. Trans. R. Soc. Lond. B. Biol. Sci. 357, 16951708.

Sherman, S.M., Guillery, R.W., 2005. Exploring the Thalamus and its Role in Cortical Function. The MIT press, Cambridge (USA).

Sherman, S.M., 2007. The thalamus is more than just a relay. Curr. Opin. Neurobiol. 17, 417-422.

Shipp, S., Zeki, S., 1989. The organization of connections between areas V5 and V1 in macaque monkey visual cortex. Eur. J. Neurosci. 1, 309-332.

Shipp, S., Blanton, M., Zeki, S., 1998. A visuo-somatomotor pathway through superior parietal cortex in the macaque monkey: cortical connections of areas V6 and V6A. Eur. J. Neurosci. 10, 3171-3193.

Shipp, S., 2003. The functional logic of cortico-pulvinar connections. Philos. Trans. R. Soc. Lond. B. Biol. Sci. 358, 1605-1624.

Smiley, J.F., Hackett, T.A., Ulbert, I., Karmas, G., Lakatos, P., Javitt, D.C., Schroeder, C.E., 2007. Multisensory convergence in auditory cortex, I. Cortical connections of the caudal superior temporal plane in macaque monkeys. J. Comp. Neurol. 502, 894-923.

Sparks, D.L., 2002. The brainstem control of saccadic eye movements. Nat. Rev. Neurosci. 3, 952-964

Sperdin, H., Cappe, C., Foxe, J.J., Murray, M.M., 2009. Early, low-level auditorysomatosensory multisensory interactions impact reaction time speed. Front. Integr. Neurosci. 3, 2. doi:10.3389/neuro.07.002.2009.

Spinelli, D.N., Starr, A., Barrett, T.W., 1968. Auditory specificity in unit recordings from cat's visual cortex. Exp. Neurol. 22, 75-84.

Stein, B.E., Meredith, M.A., 1993. The Merging of the Senses. MIT Press, Cambridge, Mass.

Stein, B.E., 1998. Neural mechanisms for synthesizing sensory information and producing adaptive behaviors. Exp. Brain Res. 123, 124-135.

Stepniewska, I., 2004. The Pulvinar Complex. The Primate Visual System. CRC Press, (Chapter 3). pp. 53-79.

Tanné-Gariépy, J., Rouiller, E.M., Boussaoud, D., 2002. Parietal inputs to dorsal versus ventral premotor areas in the macaque monkey: evidence for largely segregated visuomotor pathways. Exp. Brain Res. 145, 91-103.

Taktakishvili, O., Sivan-Loukianova, E., Kultas-Ilinsky, K., Ilinsky, I.A., 2002. Posterio parietal cortex projections to the ventral lateral and some association thalamic nuclei in Macaca mulatta. Brain Res. Bull. 59, 135-150.

Van Horn, S.C., Sherman, S.M., 2004. Differences in projection patterns between large and small corticothalamic terminals. J. Comp. Neurol. 475, 406-415.

Wang, Y., Celebrini, S., Trotter, Y., Barone, P., 2008. Visuo-auditory interactions in the primary visual cortex of the behaving monkey: electrophysiological evidence. BMC Neurosci. 9, 79.

Watanabe, J., Iwai, E., 1991. Neuronal activity in visual, auditory and polysensory areas in the monkey temporal cortex during visual fixation task. Brain Res. Bull. 26 (4), 583-592.

Watkins, S., Shams, L., Tanaka, S., Haynes, J.D., Rees, G., 2006. Sound alters activity in human V1 in association with illusory visual perception. Neuroimage $31,1247-$ 1256.

Welch, R.B., Warren, D.H., 1986. Intersensory interactions. In: Thomas, J.P. (Ed.) Handbook of Perception and Human Performance, vol. I. Sensory Processes and Perception. New York, Wiley. pp. 25-21-25-36.

Wise, S.P., Boussaoud, D., Johnson, P.B., Caminiti, R., 1997. Premotor and parietal cortex: corticocortical connectivity and combinatorial computations. Annu. Rev. Neurosci. 20, 25-42.

Yirmiya, R., Hocherman, S., 1987. Auditory- and movement-related neural activity interact in the pulvinar of the behaving Rhesus monkey. Brain Res. 402, 93-102. 